\title{
The American Phytopathological Society Elected and Appointed Officers, Representatives, and Committees for 2005
}

Council

President: J. D. MacDonald

President-Elect: J. H. Andrews

Immediate Past President: G. C. Bergstrom

Vice President: J. E. Leach

Secretary: D. T. Beadle

Treasurer: E. L. Stromberg

Editor-in-Chief, Phytopathology: C. C. Mundt

Editor-in-Chief, Plant Disease: K. L. Bowen

Editor-in-Chief, Molecular Plant-Microbe Interactions: J. Stougaard

Editor-in-Chief, Phytopathology News: M. L. Daughtrey

Editor-in-Chief, APS Press: R. C. Gergerich

Senior Councilor-at-Large: M. A. Ellis

Intermediate Councilor-at-Large: A. H. Tally

Junior Councilor-at-Large: B. J. Christ

Councilor of the Caribbean Division: J. K. Brown

Councilor of the North Central Division: R. D. Martyn

Councilor of the Northeastern Division: M. T. McGrath

Councilor of the Pacific Division: G. G. Grove

Councilor of the Potomac Division: T. A. Evans

Councilor of the Southern Division: G. J. Holmes

Ex officio: APS Foundation Rep.: D. E. Mathre, Executive VP: S. C. Nelson

\section{Representatives}

American Association for the Advancement of Science: D. G. Gilchrist

American Institute of Biological Sciences: J. E. Leach

American Type Culture Collection: G. H. Lacy

Coalition for Funding Agricultural Research: J. L. Sherwood

Council for Agricultural Science \& Technology: T. B. Sutton

International Society for Aboriculture: D. Neely

International Society for Plant Pathology Chair: A. M. Alvarez. G. S. Abawi,

R. A. Bennett, K. F. Cardwell, L. V. Madden, J. W. Moyer, C. C. Mundt,

L. Sequeira, P. S. Teng, A. H. van Bruggen, R. S. Zeigler

International Union of Microbiological Societies: C. J. Baker

Mycological Society of America: C. M. Stiles

Nat'l Council for Science and the Environment: K. L. Pernezny

Society of Environmental Toxicology and Chemistry: M. Simini

Society of Nematologists: E. L. Davis

APS Historian: P. D. Peterson

APS Foundation, Board of Directors

Chair: D. E. Mathre. Vice Chair: A. R. Chase. Secretary: W. E. Fry. Treasurer: E. L. Stromberg. D. A. Cooksey, C. J. D'Arcy, M. L. Gleason, G. L. Schumann, Ex officio: D. G. Schmale

Office of Electronic Communications (OEC) Board

Director: J. B. Ristaino. APSnet Feature Editor: D. M. Eastburn. D. T. Beadle, C. T. Bull, W. Chun, G. Hughes, K. C. Steddom, E. C. Tedford

\section{Office of International Programs (OIP) Advisory Board}

Director: R. C. Ploetz. Immediate Past Director: G. S. Abawi. M. Babadoost, J. Fletcher, N. Grunwald, D. P. Maxwell, S. A. Miller, J. R. Steadman, Ex officio: W. E. Fry

Education Committee: Chair: J. E. Bowman, B. Guo, D. M. Huber, S. L. Von Broembsen

Policies and Programs: Chair: R. S. Zeigler. B. K. Duffy

Research: Chair: N. A. Altier, J. K. Brown, C. A. Clark, J. P. Wilson

Service: Chair: S. A. Miller. M. Babadoost, C. Estevez deJensen, L. Marques

\section{Office of Industry Relations (OIR) Board}

Director: H. V. Morton. Immediate Past Director: C. M. Becker. L. Fought, M. D. Lees, G. P. Munkvold, B. D. Olson, B. Rao, C. T. Schiller, D. L. Thomas, Industry Liaison: M. R. Schwarz, Private Practice Liaison: W. T. Cobb, Sus. Assoc. Liaison: K. S. Arthur
Office of Public Affairs and Education (OPAE) Advisory Board

Director: D. J. Jardine. Immediate Past Director: Communications Coordinator: J. P. Damicone. K. F. Cardwell, C. R. Curtis, M. A. Draper, J. E. Partridge, A. H. Tally, PPB Chair: J. L. Sherwood, APSnet Feature Editor: D. M. Eastburn, Immediate Past President: G. C. Bergstrom

Public Policy Board (PPB)

Chair: J. L. Sherwood. J. H. Andrews, N. B. Carroll, S. M. Coakley, J. Fletcher, S. E. Gold, J. E. Leach, J. D. MacDonald, D. C. McGee, J. R. Steadman, R. E. Stuckey, Ex officio: D. J. Jardine, K. A. Eversole

Scientific Programs Board

Director: E. N. Rosskopf. Section Chairs: S. T. Adkins, R. M. Bostock, C. T. Bull, S. M. Douglas, M. L. Elliott, J. E. Lundquist.. Workshops Chair: D. G. Fernando. J. H. Andrews, M. A. Ellis, J. E. Leach, G. L. Schumann

APS Publications Board

Chair: K. L. Bowen. D. M. Benson, M. L. Daughtrey, R. C. Gergerich, C. C. Mundt, J. B. Ristaino, G. L. Schumann, J. Stougaard

APS Press

Editor-in-Chief: R. C. Gergerich. Senior Editors: A. M. Alvarez, C. T. Bull, G. Hughes, R. R. Martin, S. A. Miller, T. C. Paulitz, M. Putnam, C. P. Woloshuk, Ex officio: E. L. Stromberg

Biological \& Cultural Tests: Senior Editor: S. C. Bost. Section Editors: P. Bertrand, M. C. Black, P. M. Brannen, W. H. Elmer, D. E. Hershman, J. A. Lamondia, M. C. Matheron, M. A. Newman, N. A. Tisserat, J. A. Wrather

Fungicide and Nematicide Tests: Senior Editor: D. S. Egel. Section Editors: M. Babadoost, C. M. Becker, J. W. Buck, W. O. Cline, D. R. Cooley, A. Henn, K. L. Everts, R. C. Kemerait, W. Kirk, N. Lalancette, D. B. Langston, J. S. Miller, P. Phipps, J. R. Rich, A. C. Schilder, K. W. Seebold, C. Strausbaugh, W. Uddin

Illustrations of Plant Pathogens and Diseases: Chair: T. C. Paulitz. C. S. Rothrock, P. M. Sforza, S. L. Giammaria

Phytopathological Classics: Chair: P. D. Peterson. K. G. Scholthof

Standardization of Common Names of Plant Diseases: Chair: P. F. Bertrand

Editors of Molecular Plant-Microbe Interactions

Editor-in-Chief: J. Stougaard. Senior Editors: G. Bloemberg, S. P. DineshKumar, P. Dodds, O. Geiger, G. Gheysen, F. Govers, M. Grant, M. Parniske, P. C. Ronald, H. B. Scholthof, J. Sweigard, M. Udvardi, L. L. Walling, F. F. White. Indexing Editor: T. Kommedahl. Associate Editors: J. Bakker, A. Becker, L. M. Ciuffetti, J. N. Culver, T. P. Denny, K. F. Dobinson, X. Dong, M. Gijzen, S. Gold, E.-P. Journet, H. Kouchi, Y. Kubo, K. N. Lambert, G. J. Loake, S. A. Lommel, I. M. Lopez-Lara, E. Martinez-Romero, B. Mauch-Mani, J. M. McDowell, Y. Moenne-Loccoz, M. B. Mudgett, P. F. Palukaitis, U. Paszkowski, K. Pawlowski, J. M. Salmeron, F. Sanchez, K. Scholthof, S. C. Somerville, K. Szczyglowski, N. J. Talbot, B. Thomma, I. Toth, J. Verchot-Lubicz, S. A. Whitham

\section{Editors of Phytopathology}

Editor-in-Chief: C. C. Mundt. Senior Editors: J. C. Correll, M. J. Davis, S. Gold, B. A. Jaffee, L. L. Kinkel, R. P. Larkin, F. N. Martin, M. G. Milgroom, T. E. Mirkov, B. H. Ownley, H. Scherm, D. C. Stenger, J. P. Wilson. Associate Editors: J. E. Adaskaveg, J. Borneman, X. Chen, B. Ding, S. W. Ding, L. R. Gale, K. A. Garrett, J. S. Gerik, A. E. Glenn, R. C. Grube, N. Grunwald, J. S. Hartung, C. R. Howell, P. S. McManus, D. N. McRoberts, B. McSpadden Gardener, J. F. Murphy, E. B. Nelson, T. Powers, D. M. Rizzo, J. A. Rollins, W. Schneider, K. Scholthof, C. D. Smart, G. W. Sundin, L. Tredway, W. Turechek, J. E. Yuen

\section{Editors of Plant Disease}

Editor-in-Chief: K. L. Bowen. Senior Editors: W. Chun, M. L. Gleason, F. A. Gray, A. P. Keinath, R. C. Larsen, M. T. McGrath, G. Newcombe, J. W. Pscheidt, J. C. Rupe, L. W. Timmer, J. Y. Uchida. Focus Editor: T. Kommedahl. Feature Editor: M. P. McMullen. Disease Notes Assigning Editor: R. E. Baird. Associate Editors: R. Creamer, E. D. De Wolf, A. Gould, G. Holcomb, D. A. Inglis, W. Kirk, S. T. Koike, B. D. McCallum, M. T. Mmbaga, H. R. Pappu, A. T. Ploeg, Z. A. Pretorius, M. G. Redinbaugh, K. W. Seebold Jr., W. Uddin, R. R. Walcott, A. Westphal, F. Workneh, P. J. Zambino, N. K. Zidack 
Editors of Plant Health Progress

Editor-in-Chief: D. M. Benson. Senior Editors: L. Fought, J. E. Funderburk, R. L. Gilbertson, S. B. Martin, R. N. Raid. Ex officio: D. M. Eastburn, R. C. Gergerich

\section{Editors of Plant Health Instructor / APSnet Education Center}

Editor-in-Chief: G. L. Schumann. Senior Editors: E. J. Braun, A. Gould, N. Grunwald, M. A. Langham, J. L. Parke, D. F. Ritchie, M. A. Sulzinski, E. C. Tedford, L. J. Vaillancourt. APSnet Feature Editor: D. M. Eastburn

\section{Phytopathology News Advisory}

Editor-in-Chief: M. L. Daughtrey. A. Grybauskas, M. Lorito, K. Y. O. Osikanlu, A. H. Tally

\section{Society Internal Relations Committees}

Awards and Honors: Chair: T. R. Gottwald.. Vice Chair: L. S. Thomashow Immediate Past Chair: M. L. Powelson. M. E. Daub, M. Dickman, S. A. Ghabrial, C. E. Windels

Committee on Committees: Chair: M. A. Ellis. A. H. Tally, B. J. Christ

Financial Advisory: Chair: E. L. Stromberg. J. H. Andrews, M. A. Ellis, J. E. Leach, J. D. MacDonald, R. C. Gergerich, R. D. Martyn

Headquarters Operations: Chair: G. C. Bergstrom, J. H. Andrews, J. D. MacDonald, E. L. Stromberg, AACC representatives, Ex officio: S. C. Nelson

\section{Society General Policies Committees}

Biotechnology Impact Assessment: Chair: Y. Jia. Immediate Past Chair: A. O. Charkowski. Vice Chair: TBD. M. A. Bhatti, R. Hull, F. W. Nutter, Jr., S. D. Soby

Collections and Germplasm: Chair: B. J. Goates. Immediate Past Chair: F. M. Dugan. Vice Chair: J. Chen. P. A. Berland, X. Chen, K. M. Crosby, J. A. Thies, C. E. Thomas, A. H. Yahyaoui

Early Career Professionals: Chair: S. J. Vasquez, Vice Chair: J. K. O’Mara, M. C. Becktell, C. T. Bull, N. J. Catlin, A. E. Glenn, B. K. ScholzSchroeder, N. R. Walker, Y. Zhao

Extension: Chair: G. M. Leavitt. Immediate Past Chair: G. G. Grove. Vice Chair: L. J. Giesler. E. B. Adams, C. A. Bradley, A. K. Hagan, T. W. Hall, C. R. Hollingsworth, G. J. Holmes, Y. Luo, D. K. Malvick, J. C. Mertely, N. A. Mitkowski, K. Ong, J. P. Stack, S. J. Vasquez

Graduate Student: Chair: D. G. Schmale. Immediate Past Chair: P. D. Esker. Vice Chair: TBD. R. M. Andrie, T. A. Bowers, L. N. Caldwell, N. J. Catlin, K. D. Cox, M. Diagne, C. Lapaire Harmon, A. Kilaru, K. M. Linholm, S. C. Savchuk, M. VanWyk

Industry: Chair: M. R. Schwarz. Immediate Past Chair: R. Soufi. Vice Chair: B. D. Olson. J. Mike Bonman, J. V. Florentine, J. A. Frank, Z. KanaanAtallah, R. T. Lartey, M. R. Miles, G. P. Munkvold, Z. Shi, M. G. Tiffany, P. Warrior

Joint Committee of Women in Plant Path \& Cultural Diversity: Chair: C. Yandoc. Immediate Past Chair: D. G. Fernando. Vice Chair: TBD. M. E. Ambroson, A. A. Bacetty, C. T. Bull, C. Cowger, L. K. Hawkins, P. Hughes-Watson, N. I. Khan, M. T. Mmbaga, Y. Zhang

Placement: Chair: A. DeMarsay. Immediate Past Chair: A. A. Collins. Vice Chair: J. E. Creasap. B. J. Bush, K. W. Campbell, J. F. Davey, E. N. Rosskopf, L. Ines Santamaria, T. V. Taylor, H. E. Velez

Private Practice: Chair: C. L. Kohls. Immediate Past Chair: A. R. Chase. Vice Chair: TBD. S. J. Day, C. S. Thomas

Regulatory Plant Pathology: Chair: P. I. Lewis. Immediate Past Chair: T. N. Boratynski. Vice Chair: G. L. Peterson. S. S. Hurtt, M. J. Jones, R. F. Lee, V. S. Malik, N. K. Osterbauer, E. V. Podleckis, K. L. Smith, X. Sun, R. E. Ykema

Teaching: Chair: G. W. Hudler. Immediate Past Chair: E. J. Braun. Vice Chair: TBD. L. P. Berkett, R. G. Bhat, R. B. Carroll, D. M. Eastburn, R. C. Gergerich, S. K. Gremillion, M. B. Riley, R. W. Schneider, C. M. Stiles

Youth Program: Chair: S. D. Callahan Useman. Immediate Past Chair: C. A. Jasalavich. Vice Chair: TBD. J. H. Brock, S. M. Douglas, B. A. Edmunds, W. H. Elmer, J. E. Painter, M. J. Ryba-White, K. W. Seebold, Jr., P. W. Tooley

\section{Subject Matter Committees}

Bacteriology: Chair: S. Y. He. Immediate Past Chair: G. A. Beattie. Vice Chair: D. Y. kobayashi. A. J. Bogdanove, M. L. Brodhagen, A. O. Charkowski, W. Chun, W. C. Fuqua, J. B. Jones, L. M. Keith, I. Lee, K. L. Newman, J. L. Norelli, N. W. Schaad, G. W. Sundin, S. B. Von Bodman

Biochemistry, Physiology \& Molecular Biology: Chair: L. J. Vaillancourt. Immediate Past Chair: C. B. Lawrence. Vice Chair: TBD. J. J. Choi, L. M. Ciuffetti, M. B. Dickman, J. E. Flaherty, Y. Jia, J. M. McDowell, W. Shim, C. D. Smart, D. S. Warkentin, R. P. Wise, C. P. Woloshuk

Biological Control: Chair: R. P. Larkin. Immediate Past Chair: B. J. Jacobsen. Vice Chair: A. A. Collins. H. K. Abbas, P. D. Adams, P. D. Kharbanda, S. Kiewnick, D. C. Sands, D. A. Schisler, J. P. Stack, L. S. Thomashow, D. M. Weller, C. Yandoc
Chemical Control: Chair: R. C. Kemerait. Immediate Past Chair: K. W. Seebold, Jr. Vice Chair: TBD. S. A. Alexander, CJ. P. Damicone, M. FR. Khan, C. L. Kohls, C. J. Mickler, B. D. Olson, G. L. Schuster, K. L. Smith, R. Soufi, D. C. Thompson, L. E. Zang

Crop Loss Assessment and Risk Evaluations: Chair: C. A. Hollier. Immediate Past Chair: D. A. Shah. Vice Chair: TBD. G. Hughes, D. L. Long, S. J. Pethybridge, D. G. Schmale, W. W. Turechek, C. R. Wilson

Diagnostics: Chair: J. L. Chaky. Immediate Past Chair: B. D. Ambruzs. Vice Chair: M. Babadoost, T. D. Blunt, L. G. Brown, S. M. Douglas, M. Hanson, J. C. Jacobi, D. K. Malvick, R. T. McMillan, J. L. O’Mara, G. E. Ruhl, K. L. Snover-Clift, C. L. Sutula

Diseases of Ornamental Plants: Chair: S. N. Wegulo. Immediate Past Chair: C. Hong. Vice Chair: W. E. Copes. J. M. Byrne, G. T. Church, B. A. Edmunds, J. R. Hartman, S. N. Jeffers, A. D. Martinez-Espinoza, R. J. McGovern, R. P. Mulrooney, S. G. P. Nameth, M. J. Pearce, B. Rao, B. B. Reddick, C. Y. Warfield, R. L. Wick, J. Williams-Woodward

Environmental Quality \& Plant Health: Chair: C. R. Krause. Immediate Past Chair: M. Simini. Vice Chair: TBD. M. A. Bhatti, M. S. Krause

Epidemiology: Chair: E. D. De Wolf. Immediate Past Chair: F. W. Nutter, Jr. Vice Chair: R. D. Magarey. C. Cowger, P. D. Esker, K. A. Garrett, C. R. Hollingsworth, R. Jaime-Garcia, R. Khangura, M. Nita, P. M. Sforza, G. E. Shaner, K. M. Tubajika

Forest Pathology: Chair: P. J. Zambino. Immediate Past Chair: S. D. Cohen. Vice Chair: P. Bonello. D. N. Appel, C. L. Ash, K. S. Camilli, D. J. Collins, L. Englander, E. Frankel, J. A. Hoff, M. Kim, G. Laflamme, B. D. Moltzan, S. W. Oak, C. G. Shaw, III, J. K. Stone

Genetics: Chair: R. L. Bowden. Immediate Past Chair: S. Kamoun. Vice Chair: N. Grunwald. X. Chen, K.-R. Chung, M. Garcia-Pedrajas, S. D. Harris, C. Khang, S. J. Klosterman, Y.-H. Lee, F. N. Martin, B.G. Turgeon, J.-R. Xu, K. A. Zeller

Host Resistance: Chair: M. G. Redinbaugh. Immediate Past Chair: M. R. Miles. Vice Chair: C. R. Grau. R. G. Bhat, J. M. Bonman, M. L. Carson, B. Guo, C. B. Hill, K. M. Linholm, A. Mengistu, B. J. Steffenson, J. A. Thies, C. M. Vera Cruz, A. H. Yahyaoui, X. Zheng

Integrated Pest Management: Chair: T. M. Momol. Immediate Past Chair: D. S. Egel. Vice Chair: L. J. Gutierrez. K. L. Everts, W. McFadden-Smith, B. J. Messenger, P. D. Roberts, S. F. Shamoun

Mycology: Chair: C. L. Harmon. Immediate Past Chair: S. B. Goodwin. Vice Chair: TBD. D. M. Beyer, R. Blanco Prieto, T. E. Chase, K. D. Cox, J. C. Dianese, D. M. Geiser, D. A. Glawe, G. W. Hudler, J. L. Kerrigan, M. E. Palm, S. A. Rizvi, M. Skaria

Mycotoxicology: Chair: R. L. Brown. Immediate Past Chair: G. Kuldau. Vice Chair: TBD. C. W. Bacon, B. J. Bush, A. M. Fakhoury, D. L. Funnell, T. J. Michailides, R. H. Proctor, B. M. Pryor

Nematology: Chair: J. B. Kotcon. Immediate Past Chair T. L. Kirkpatrick. Vice Chair: TBD. A. A. Bacetty, J. Desaeger, R. A. Dunn, J. L. Padgham, M. D. Petrillo, B. S. Sipes, G. L. Tylka, K.-H. Wang

Pathogen Resistance: Chair: M. T. McGrath. Immediate Past Chair: K. L. Stevenson. Vice Chair: TBD. R. P. Kaiser, W. D. Koeller, P. I. Lewis, H. V. Morton, G. Olaya, G. Schnabel, W. F. Wilcox, F. P. Wong

Phyllosphere Microbiology: Chair: G. Y. Yuen. Immediate Past Chair: W. F. Mahaffee. Vice Chair: TBD. M. T. Brandl, T. J. Burr, V. Toussaint

Plant Pathogen and Disease Detection: Chair: R. T. McMillan. Immediate Past Chair: P. Ellis. Vice Chair: R. R. Martin. P. H. Berger, J. D. Dominiak, R. Frederick, L. Levy, D. G. Luster, S. K. Marquardt, V. A. Mavrodieva, J. H. McBeath, A. Rowhani, M. G. Tiffany, I. E. Tzanetakis

Postharvest Pathology: Chair: J. L. McEvoy. Vice Chair: J. E. Adaskaveg. J. A. Bartz, B. D. Bruton, A. Cochran, W. S. Conway, G. J. Holmes, W. J. Janisiewicz, J. Mercier, D. B. Prusky, P. L. Sholberg, J. L. Smilanick, D. Sugar, E. C. Tedford, C. Xiao, J. Zhang

Seed Pathology: Chair: B. L. Randall-Schadel. Immediate Past Chair: L. M. Shepherd. Vice Chair: W. L. Wiebe. K. S. Arthur, S. Autrey, W. O. Bliss, C. C. Block, D. O. Burrell, J. L. Daniels, K. D. Gwinn, P. T. Himmel, H. Koenraadt, G. L. Lamka, M. Lusso, D. C. McGee, A. Tayfour, E. Vivoda, R. R. Walcott

Soil Microbiology and Root Diseases: Chair: B. Spakes Richter. Immediate Past Chair: J. L. Parke. Vice Chair: TBD. J. C. Bienapfl, E. Blume, L. E. Hanson, J. Hao, D. M. Huber, C. D. Lewis, S. Sanogo, K. L. Schroeder, D. M. Weller

Tropical Plant Pathology: Chair: F. M. Ochoa. Immediate Past Chair: L. A. Calvert. Vice Chair: R. H. Brlansky. A. Kilaru, J. O. Kuti, S. M. Njoroge, R. A. Valverde, J. A. Ventura, J. P. Wilson

Turfgrass Pathology: Chair: P. F. Harmon. Immediate Past Chair: L. P. Tredway. Vice Chair: TBD. M. J. Boehm, L. L. Burpee, P. H. Dernoeden, J. E. Kaminski, E. B. Nelson, H. C. Wetzel

Virology: Chair: S. T. Adkins. Immediate Past Chair: J. K. Brown. Vice Chair: TBD. J. D. Bradshaw, R. C. French, R. W. Hammond, S. F. Hanson, A. M. Harness, M. E. Hilf, S. Hogenhout, A. M. Idris, D. Robertson, W. Schneider, D. C. Stenger 